\title{
A cohort study evaluating paraaortic lymphadenectomy in endometrial cancer
}

\author{
HAIYAN ZHANG, ZHI ZUO, YE WANG, LI WANG and ZHILING ZHU \\ Department of Gynecology, Obstetrics and Gynecology Hospital of Fudan University, \\ Shanghai 200011, P.R. China
}

Received May 31, 2012; Accepted August 9, 2012

DOI: 10.3892/ol.2012.919

\begin{abstract}
The current study sought to assess the role of paraaortic lymphadenectomy (LNE) in females with endometrial cancer. A retrospective analysis of patients diagnosed with endometrial cancer of stage IA to II preoperatively, between 2009 and 2011 was conducted. Patients were included who had suffered from endometrial cancer without preoperative adjuvant therapy and who underwent hysterectomy plus systematic pelvic LNE and paraaortic LNE by laparoscopy or laparotomy. A total of 54 patients who underwent surgery for preoperative endometrial cancer were selected. All patients underwent LNE. The incidences of pelvic and paraaortic lymph node metastases were $11.1 \%(6 / 54)$ and $7.4 \%(4 / 54)$, with a total positive lymph node rate of $14.8 \%(8 / 54)$. In addition, among the 8 positive cases, 5 patients underwent laparotomy and 3 underwent laparoscopy; 3 cases were classified as stage I and 5 as stage II preoperatively. Of these, 7 patients were identified with pathology-related risk factors, including low differentiation or clear cell adenocarcinoma postoperatively. Discordance of pathological differentiation between the pre- and postoperative stages reached $57.1 \%$ (4/7). The results revealed the high occurrence of positive lymph nodes in endometrial cancer which demonstrate the importance of systematic LNE. Additonally, no severe complications were caused by LNE besides lymph cysts. In summary, it is neccesary to perform LNE, particularly the removal of the paraaortic lymph node, in patients with endometrial cancers in order to improve postoperative therapy. Laparoscopy has similar surgical effects as laparotomy, but has a number of advantages.
\end{abstract}

Correspondence to: Professor Zhiling Zhu, Department of Gynecology, Obstetrics and Gynecology Hospital of Fudan University, 419 Fangxie Road, Shanghai 200011, P.R. China E-mail: zhilingzhu888@126.com

Abbreviations: LNE, lymphadenectomy; PLN, pelvic lymph nodes; PAN, paraaortic lymph nodes

Key words: lymphadenectomy, endometrial cancer, paraaortic

\section{Introduction}

Endometrial cancer is the most common gynecological malignancy in developed countries (1). Standard management includes the surgical removal of the uterus, Fallopian tubes and ovaries (2). Metastasis to the pelvic lymph nodes (PLN) or paraaortic lymph nodes (PAN) is one of the most significant factors associated with the determination of the clinical stage and prognosis of endometrial cancer. The reported frequency of PLN and/or PAN metastasis ranges from 5 to $20 \%$ (3-6) and patients with retroperitoneal lymph node metastasis are classified as stage IIIC. Thus, it is important to evaluate the presence or absence of PAN metastasis when surgically staging this disease and predicting its prognosis.

However, in previous years, the issue of the therapeutic effect of lymphadenectomy (LNE) in endometrial cancer has been debated. LNE and PAN dissection are not routine operative procedures at present. Certain authors view it as a curative approach in retrospective studies, while others consider the value of LNE as a purely diagnostic measure and others doubt the therapeutic significance of this surgery (7-9). However, there is an agreement that pelvic and/or PAN involvement worsens the prognosis of endometrial carcinoma significantly (10-13). Knowledge of lymph node involvement or other extrauterine spread generally affects postoperative treatment in the form of irradiation or chemotherapy. It appears unreasonable that systematic LNE does not include regional lymph node dissection, involving either the biopsy of enlarged nodes only, selective nodal sampling, or complete LNE.

To date, there are no studies on LNE in patients with endometrial cancer from China, particularly the paraaortic LNE by laparoscopy. Our hospital is one of the first centers in China to initiate the use of laparoscopy in the gynecological field, particularly in gynecological tumors. The patients with endometrial cancer who underwent hysterectomy and systematic LNE plus paraaortic LNE by laparoscopy or laparotomy were retrospectively analyzed. The diagnostic significance of paraaortic LNE for patients with or without intermediate- or high-risk endometrial cancer was identified, based on the hypothesis that the PAN area is susceptible to recurrence if omitted from surgery and metastasis to the PAN area influences adjuvant treatment. It is not possible to accurately stage endometrial malignancies if the PAN area is neglected, and this is likely to aggravate radiotherapy. Therefore, this study 
Table I. Total LN and positive LN counts.

\begin{tabular}{|c|c|c|c|c|c|}
\hline & \multirow{2}{*}{$\begin{array}{c}\text { Number of } \\
\text { cases }\end{array}$} & \multicolumn{2}{|c|}{ Total LN count, average $(\%)$} & \multicolumn{2}{|c|}{ Positive LN count } \\
\hline & & Pelvic & Paraaortic & Pelvic & Paraaortic \\
\hline \multicolumn{6}{|l|}{ Stage } \\
\hline IA & $8(\mathrm{~T})+24(\mathrm{~L})$ & $644(20.1)$ & $129(4.0)$ & & \\
\hline IB & $6(\mathrm{~T})+4(\mathrm{~L})$ & $228(22.8)$ & $43(4.3)$ & & \\
\hline II & $2(\mathrm{~T})+1(\mathrm{~L})$ & $64(21.3)$ & $11(3.7)$ & & \\
\hline IIIA & $1(\mathrm{~L})$ & $17(17)$ & $2(2)$ & & \\
\hline IIIC & $5(\mathrm{~T})+3(\mathrm{~L})$ & $159(19.8)$ & $22(2.8)$ & & \\
\hline \multicolumn{6}{|c|}{ Surgery pathway } \\
\hline Laparotomy & 21 & $453(21.6)$ & $72(3.4)$ & 7 & 5 \\
\hline Laparoscopy & 33 & $659(20.0)$ & $135(4.1)$ & 9 & 2 \\
\hline Total & & & & 16 & 7 \\
\hline
\end{tabular}

LN, lymph node; T, laparotomy; L, laparoscopy.

intends to analyze the positive rate of PAN metastasis in patients with endometrial cancer in our hospital and compare the difference between laparoscopy and laparotomy. The aim of the current study was to investigate the importance of paraaortic LNE in endometrial cancer and the advantage of laparoscopy over laparotomy.

\section{Patients and methods}

A total of 54 patients were selected who had undergone hysterectomy plus systematic pelvic LNE and paraaortic LNE by laparoscopy or laparotomy in the previous two years, dating from 12/1/2009 to 23/5/2011, in the Obstetrics and Gynecology Hospital of Fudan University (Shanghai, China). This study was approved by the Ethics Committee of the Obstetrics and Gynecology Hospital of Fudan University. Written informed consent was obtained from all patient's family. In total, 33 cases were laparoscopic and 21 cases were laparotomic. Lymph node count and status, escalation of stage, histology type, duration of surgery, estimated blood loss, peri- and postoperative complications, postoperative hospitalization and recovery and history of irregular menstral cycle and menopausal vaginal bleeding were recorded. All surgical procedures were performed by experienced gynecological oncologists at the Obstetrics and Gynecology Hospital of Fudan University. All diagnoses of cancer were histologically confirmed. Furthermore, the duration of surgery and blood loss were evaluated to judge the feasibility of the paraaortic LNE and compare the two surgery pathways technically. Data were collected in an intent to treat manner and then analyzed using SAS 9.2 (SAS Institute, Cary, North Carolina). The t- and wilcox-tests were used to assess the differences between the laparoscopy and laparotomy cohorts.

\section{Results}

Lymph node count and status. We calculated the total lymph node count in various stages and various surgery pathways. The average paraaortic lymph node count of the laparotomy
Table II. Positive LN status.

\begin{tabular}{lcc}
\hline PAN status & PLN status & Incidence $(\%)$ \\
\hline+ & + & $2 / 54(3.7)$ \\
+ & - & $4 / 54(7.4)$ \\
- & + & $2 / 54(3.7)$ \\
Total & & $8 / 54(14.8)$ \\
\hline
\end{tabular}

LN, lymph node; PAN, paraaortic lymph nodes; PLN, pelvic lymph nodes.

group was 3.14, while the laparoscopy group was 2.40 . No statistical difference was observed between the two groups of the PANs. The lymph node status was also analyzed. A total of 8 cases (14.8\% of patients) were identified as positive for either PAN or PLN metastasis. No other high-risk factors were identified in $4 / 8$ patients prior to surgery, with the exception of invasive depth $>1 / 2$ myometrial layer (19 patients were identified with invasive depth $>1 / 2$ myometrial layer in total). Low differentiation was identified by curettage in 2 patients. The final 2 patients were free of high-risk factors. A positive lymph node status was not predictable based on the preoperative status as there is no definite method for predicting lymph node metastasis, therefore systematic pelvic LNE and paraaortic LNE should be performed to make staging more precise and to guide the postoperative therapy, particularly radiation (Tables I and II).

Pre- and postoperation stage. According to the FIGO guidelines, the stage of endometrial cancer is mainly categorized by pathological status. Therefore, occasionally there is an elevation of stage following surgery, as the status of the lymph node cannot be precisely predicted prior to surgery. It has been reported that a substantial number of patients with grade 1 endometrial cancer based on pre- and intraoperative assessments have a higher grade of disease at final pathology. In the 
Table III. Postoperative histology distribution of the endometrial cancer.

\begin{tabular}{|c|c|c|c|c|c|}
\hline Histology & No. patients & Incidence, n (\%) & PAN, n (\%) & PAN/PLN, n (\%) & PLN, n (\%) \\
\hline \multicolumn{6}{|l|}{ Endometrioid adenocarcinoma } \\
\hline Grade 1 & 20 & $1(5)$ & $0(0)$ & $0(0)$ & $1(5)$ \\
\hline Grade 2 & 15 & $1(6.7)$ & $1(6.7)$ & $0(0)$ & $2(13.3)$ \\
\hline Grade 3 & 9 & $1(11.1)$ & $1(11.1)$ & $2(22.2)$ & $4(44.4)$ \\
\hline Serous adenocarcinoma & 5 & $1(20.0)$ & $0(0)$ & $0(0)$ & $1(25.0)$ \\
\hline Clear cell adenocarcinoma & 5 & $0(0)$ & $0(0)$ & $0(0)$ & $0(0)$ \\
\hline
\end{tabular}

PAN, paraaortic lymph nodes; PLN, pelvic lymph nodes.

Table IV. Average blood loss and duration of surgery.

\begin{tabular}{|c|c|c|c|c|c|}
\hline Characteristic & $\begin{array}{l}\text { Number } \\
\text { of cases }\end{array}$ & $\begin{array}{c}\text { Average } \\
\text { blood loss }(\mathrm{ml})\end{array}$ & P-value & $\begin{array}{c}\text { Average operating } \\
\text { time }(\mathrm{h})\end{array}$ & P-value \\
\hline \multicolumn{6}{|l|}{ Stage } \\
\hline I & 32 & 342.19 & 0.2918 (t-test) & 3.92 & 0.6823 (t-test) \\
\hline II, III & 22 & 459.09 & 0.4278 (Wilcoxon test) & 4.04 & 0.4227 (Wilcoxon test) \\
\hline IA & 8 & 375 & $0.6564(\mathrm{t}$-test $)$ & 3.73 & 0.6176 (t-test) \\
\hline IB & 24 & 331.25 & 0.5361 (Wilcoxon test) & 3.98 & 0.8106 (Wilcoxon test) \\
\hline \multicolumn{6}{|c|}{ Surgery pathway } \\
\hline Laparotomy & 21 & 500 & 0.1198 (t-test) & 3.90 & 0.7189 (t-test) \\
\hline Laparoscopy & 33 & 319.7 & 0.1385 (Wilcoxon test) & 4.01 & 0.9363 (Wilcoxon test) \\
\hline
\end{tabular}

current study, 20.4\% (11/54) were upgraded at final pathology with $13.0 \%(7 / 54)$ characterized as grade 2 disease and $7.4 \%$ $(4 / 54)$ characterized as grade 3 . Overall, $22.2 \%(12 / 54)$ had an advanced stage disease. A total of $42.2 \%(19 / 45)$ of patients with disease limited to the uterus had adverse features, including high risk histological variants, grade 3 disease, myometrial invasion N1/2 and/or cervical involvement. Among the 8 positive cases, 3 were classified as stage I and 5 as stage II preoperatively. Although LNE does not affect survival rate, as reported, it may identify patients with advanced disease and assist in tailoring adjuvant therapy for those with adverse risk factors. These results suggest the importance of paraaortic LNE in endometrial cancer.

Histological type. Postoperative histological analysis revealed that tumors with lower differentiation, including G3, tend to metastasize via the lymph system. Among the 8 positive cases, 7 patients were identified with pathology-related risk factors, including low differentiation or clear cell carcinoma postoperatively. Discordance of pathological differentiation between the pre- and postoperative stages reached $57.1 \%$ (4/7). Among the 5 clear cell adenocarcinomas, no incidence of positive lymph node metastasis was observed. Clear cell adenocarcinoma is considered to be invasive even in the early stages; the deviation may be demonstrated to be corrected by future studies involvoing a large number of patients. Of note, one case was staged as IA peroperatively, with no high-risk factors and was revealed to be PAN-positive but PLN-negative and G1 postoperatively (Table III).
Surgery duration and blood loss. The average surgery duration of laparotomy was $3.90 \mathrm{~h}$ and of laparoscopy was $4.01 \mathrm{~h}$. No statistical difference was observed in operating time between the two groups. The estimated blood loss was also evaluated as being on average $500 \mathrm{ml}$ for laparotomy and $319.7 \mathrm{ml}$ for laparoscopy; there was no statistical significance between the two groups. The estimated blood loss during the LNE for the majority of patients was 20-40 ml. A longer duration of surgery and more blood loss occurred only among the cases in which radical cervical resection was performed when the cervix was invaded. Stage did not affect the average blood loss or average operating time. The comparison between stage IA and IB and between I and II plus III revealed no statistical difference in blood loss or duration of surgery. The comparison of distribution of stages between the two surgery pathways revealed that the distribution has no effect (Table IV).

Complications, postoperative hospitalization and recovery. There were no clear complications caused by the surgery, with the exception of lymph cysts. The formation of lymph cysts may be partially explained by the backing pathways of lymph fluid in the pelvis being widely blocked after the LNE. The majority of patients had no symptoms and only a few patients complained of slight discomfort in the legs. The large abdominal incision created by laparotomy causes acute pain and carries a risk of a slow healing wound, particularly for obese patients. Postoperative hospitalization was much shorter in duration for the laparoscopic group, with a maximum of 7-8 days, than that of the laparotomic group, 
with a maximum of 12-15 days. Furthermore, the majority of patients in the laparoscopic group were able to dismount the bed freely 2-3 days following surgery, while in the latter group, patients were in pain, felt weak and were not willing to dismount the bed in the first week following surgery. The comparison between the two surgery pathways demonstrates that laparoscopy correlates with a shorter hospitalization and recovery period. The two surgeries have similar surgical effects for the patients when taking into consideration surgery duration, blood loss, lymph node count and status and complications.

History of irregular menstrual cycles and menopausal vaginal bleeding. The majority of patients with endometrial neoplasms had a history of irregular mentrual cycles or menopausal vaginal bleeding. In the present study, the shortest history of abnormal menstruation was one month. This 49-year-old patient was first diagnosed as stage II and was identified as G3 histologically post-surgery. This patient had no high risk factors, as assessed prior to surgery. Low differentiation of endometrial cancer cells manifests a strong malignant capacity, with minor symptoms; this may cause late stage damage of the patients, even if they are diagnosed early. The shortest duration of menopausal vaginal bleeding was 5 days. The patient had a 2-year history of hypertension and 4-year history of menopause and was diagnosed as having endometrioid cancer, characterized as stage IA and G1 following surgery. Among the non-menopausal females, $26.3 \%(5 / 19)$ had a history of irregular menstrual cycles of less than 4 months, with 1 of the 5 cases identified as PAN-postive and $47.4 \%(9 / 19)$ patients had a history of irregular menstrual cycles of less than 10 months. Among the menopausal females, $42.9 \%(15 / 35)$ had a history of menopausal vaginal bleeding for less than 3 months, with 1 of the 15 cases demonstrated to be PAN-postive. Close follow-ups and curettages of females with a history of irregular menstrual cycles and menopausal vaginal bleeding were performed, whether high risk factors were present or not.

\section{Discussion}

The metastasis to lymph nodes is a major prognostic factor among females with endometrial cancers. Therefore, careful assessment of the pathological nodal status is an integral part of the management of these patients. Histopathological examination provides the most accurate method of ascertaining the degree of metastatic spread to the lymph nodes, although the complications and practical significance of LNE remain controversial. Certain researchers have reported a low therapeutic significance of LNE, however, it causes unavoidable complications that affect the quality of life of the patients $(14,15)$, while others argue that it aids in obtaining a systematic stage for the patient, particularly from the histological aspect (16). Beside the histological type and the invasion depth the of myometrial layer, the lymph node status is extremely significant in the determination of postoperative treatment, particularly radiotherapy $(17,18)$. It has been suggested that surgery with systematic pelvic and paraaortic LNE followed by adjuvant chemotherapy improved the long-term survival rate in patients with PAN metastasis $(19,20)$. These results demonstrate that laparoscopic LNE advances minimally invasive diagnostics in endometrial cancers and may allow for a more precise prognostic assessment.

In previous years, the concept of minimally invasive surgery in the management of gynecological malignancies has gradually evovled from almost impossible to a fully realized optimal choice for a number of patients. Laparoscopy for gynecological malignancies is now used worldwide (21-23). It has been established that laparoscopy induces fewer adhesions than open dissection. The laparotomy approach subjects the patient to the morbidity associated with a large incision and consequently more complications. Recognized advantages of laparoscopy include less pain, smaller incisions, quicker recovery, shorter hospital stays, decreased blood loss, equivalent nodal counts and, in certain studies, similar survival rates. This has been confirmed in the current study.

With regard to the retrieved nodes, laparoscopic lymph node dissection has been demonstrated to be comparable to laparotomy in terms of node counts. In the current study, the mean number of nodes obtained by the laparoscopic approach was 24.1. The node count using the laparoscopic extraperitoneal approach had been shown to be comparable to that of a transperitoneal approach. This number included patients with grossly involved nodes who had a selective lymph node removal. It was also in agreement with other reports evaluating paraaortic LNE.

LNE is associated with significant operative risks in certain patients, however there were no incidences of pelvic organ trauma and little blood loss during LNE in the current study. The low incidence of postoperative complications allows prompt resumption of daily activities and the early initiation of adjuvant therapy. Lymph cysts are the most common complication post surgery, whichever surgical pathway is used, laparotomy or laparoscopy. There are no clear symptoms caused by lymph cysts. The majority of pelvic lymphocysts appear within 3 to 4 weeks following surgery, with an incidence varying from 1.5 to $25.3 \%$. In the current study, the incidence of lymphocysts was $18.5 \%$ (10/54). This difference may be due to the variable degree of complete LNE or the various methods used to perform the procedure. Although the exact mechanism of the formation of pelvic lymphocysts is not known, the majority of lymphocysts cause slight symptoms and may be treated conservatively.

In conclusion, laparotomic and laparoscopic surgeries may be successfully performed. The feasibility of performing laparoscopic LNE in endometrial cancer has been demonstrated. Laparoscopy had a smilar duration of surgery and blood loss, but had an improved influence on the patients with potentially fewer complications and faster recovery. The postoperative duration in hospital was markedly longer among the patients who underwent laparotomy. It is a safe procedure with an acceptable nodal yield, blood loss, short operating duration and low complication rate. It is effective in tailoring the field of adjuvant radiotherapy in endometrial cancers. Therefore, we consider that it is a useful tool for completing staging and confirming the adequate treatment for endometrial cancer in various stages. In the future, further experience and a longer follow-up period are required to confirm that this approach is safe and feasible. 


\section{Acknowledgements}

This study was supported by the Shanghai Natural Scientific Fund (09ZR1404700).

\section{References}

1. Jemal A, Thomas A, Murray T and Thun M: Cancer statistics, 2002. CA Cancer J Clin 52: 23-47, 2002.

2. Hacker NF: Uterine cancer. In: Practical Gynecologic Oncology. Berek JS and Hacker NF (eds). Lippincott Williams \& Wilkins, Philadelphia, pp407-455, 2000.

3. Hirahatake K, Hareyama H, Sakuragi N, Nishiya M, Makinoda S and Fujimoto S: A clinical and pathologic study on para-aortic lymph node metastasis in endometrial carcinoma. J Surg Oncol 65: 82-87, 1997.

4. Kamura T, Yahata H, Shigematsu T, et al: Predicting pelvic lymph node metastasis in endometrial carcinoma. Gynecol Oncol 72: 387-391, 1999.

5. Mundt AJ, Murphy KT, Rotmensch J, Waggoner SE, Yamada SD and Connell PP: Surgery and postoperative radiation therapy in FIGO stage IIIC endometrial carcinoma. Int J Radiat Oncol Biol Phys 50: 1154-1160, 2001.

6. McMeekin DS, Lashbrook D, Gold M, et al: Nodal distribution and its significance in FIGO stage IIIc endometrial cancer. Gynecol Oncol 82: 375-379, 2001.

7. Neubauer NL and Lurain JR: The role of lymphadenectomy in surgical staging of endometrial cancer. Int J Surg Oncol 2011: 814649, 2011.

8. Zhang H, Jia L, Zhang Q, Zhang Y, Yang X and Kong B: Therapeutic role of systematic retroperitoneal lymphadenectomy in endometrial cancer. Bull Cancer 99: 10-17, 2012.

9. Kim HS, Kim HY, Park CY, et al: Lymphadenectomy increases the prognostic value of the revised 2009 FIGO staging system for endometrial cancer: A multi-center study. Eur J Surg Oncol 38: 230-237, 2012

10. Garg G, Morris RT, Solomon L, et al: Evaluating the significance of location of lymph node metastasis and extranodal disease in women with stage IIIC endometrial cancer. Gynecol Oncol 123: 208-213, 2011.

11. Kehoe SM and Miller DS: The role of lymphadenectomy in endometrial cancer. Clin Obstet Gynecol 54: 235-244, 2011.

12. Kondalsamy-Chennakesavan S, van Vugt S, Sanday K, et al: Evaluation of tumor-free distance and depth of myometrial invasion as prognostic factors for lymph node metastases in endometrial cancer. Int J Gynecol Cancer 20: 1217-1221, 2011.
13. Park JY, Kim DY, Kim JH, Kim YM, Kim YT and Nam JH: The role of pelvic and/or para-aortic lymphadenectomy in surgical management of apparently early carcinosarcoma of uterus. Ann Surg Oncol 17: 861-868, 2010.

14. Hidaka T, Nakashima A, Shima T, Hasegawa T and Saito S: Systemic lymphadenectomy cannot be recommended for low-risk corpus cancer. Obstet Gynecol Int 2010: 490219, 2010.

15. Xu H, Chen Y, Li Y, et al: Complications of laparoscopic radical hysterectomy and lymphadenectomy for invasive cervical cancer: experience based on 317 procedures. Surg Endosc 21: 960-964, 2007.

16. Nomura H, Tsuda H, Susumu N, et al: Lymph node metastasis in grossly apparent stages I and II epithelial ovarian cancer. Int J Gynecol Cancer 20: 341-345, 2010

17. Kornovski Y and Gorchev G: Neoadjuvant chemotherapy followed by radical surgery and radiotherapy vs. pelvic irradiation in patients with cervical cancer FIGO stage IIB - IVA. J BUON 11: 291-297, 2006.

18. Rassweiler JJ, Henkel TO, Stock C, Seemann O, Frede T and Alken P: Retroperitoneal laparoscopic lymph node dissection for staging non-seminomatous germ cell tumors before and after chemotherapy. Lymphology 29: 36-44, 1996.

19. Klopp AH, Jhingran A, Ramondetta L, et al: Node-positive adenocarcinoma of the endometrium: outcome and patterns of recurrence with and without external beam irradiation. Gynecol Oncol 115: 6-11, 2009.

20. Leath CA III, Numnum TM, Kendrick JE IV, Frederick PJ, Rocconi RP, Conner MG and Straughn JM Jr: Patterns of failure for conservatively managed surgical stage I uterine carcinosarcoma: implications for adjuvant therapy. Int J Gynecol Cancer 19: 888-891, 2009.

21. Hong JH, Choi JS, Lee JH et al: Laparoscopic lymphadenectomy for isolated lymph node recurrence in gynecologic malignancies. J Minim Invasive Gynecol 19: 188-195, 2011.

22. Yoon A, Kim TJ, Lee WS, Kim BG and Bae DS: Single-port access laparoscopic staging operation for a borderline ovarian tumor. J Gynecol Oncol 22: 127-130, 2011.

23. Bae JW, Lee JH, Choi JS et al: Laparoscopic lymphadenectomy for gynecologic malignancies: evaluation of the surgical approach and outcomes over a seven-year experience. Arch Gynecol Obstet 285: 823-829, 2012. 